\title{
Wegener's granulomatosis with cardiac involvement is
}

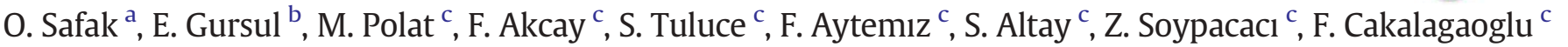 \\ ${ }^{\text {a }}$ Burdur State Hospital \\ b Canakkale State Hospital \\ c İzmir Atatürk Education and Research Hospital
}

\section{A R T I C L E I N F O}

\section{Article history:}

Received 23 March 2016

Received in revised form 26 April 2016

Accepted 29 April 2016

Available online 5 May 2016

\begin{abstract}
A B S T R A C T
Wegener's granulomatosis (granulomatosis with polyangiitis) is a form of vasculitis of small-to-medium-sized vessels and associated with diffuse anti-neutrophil cytoplasmic antibodies (cANCA). Cardiac involvement is not uncommon with $6-25 \%$ of unselected patients and up to $44 \%$ of patients with severe renal involvement. We report a 23-year-old man with Wegener's cardiomyopathy with $25 \%$ ejection fraction. The overall mortality rate of Wegener's granulomatosis with cardiac involvement has been reported to be between 15 and $45 \%$. So it is important to keep in mind that cardiac examination is a must to detect if cardiac involvement is present.

(C) 2016 The Society of Cardiovascular Academy. Production and hosting by Elsevier B.V. All rights reserved. This is

an open access article under the CC BY-NC-ND license (http://creativecommons.org/licenses/by-nc-nd/4.0/).
\end{abstract}

\section{Introduction}

Wegener's granulomatosis (granulomatosis with polyangiitis) is a form of vasculitis of small-to-medium-sized vessels and associated with diffuse anti-neutrophil cytoplasmic antibodies (cANCA). It typically affects the upper and lower airways, lungs, and kidneys. Cardiac involvement is not uncommon with $6-25 \%$ of unselected patients and up to $44 \%$ of patients with severe renal involvement. It usually presents as pericarditis, myocarditis, and aortitis, although conduction disturbances and myocardial infarction are also recognised (1). Cardiac manifestations are often not clinically apparent, but are associated with increased morbidity and mortality (2). We report a case of Wegener's cardiomyopathy with renal involvement.

\section{Case presentation}

A 23-year-old man presented with 1 month history of paroxysmal nocturnal dsypnoea, fatigue, fevers, and joint pains. On examination, he was tachypnoeic with a respiratory rate of $26 / \mathrm{min}$ and had a tachycardia of $116 / \mathrm{min}$. He was normotensive. Auscultation of his heart revealed a gallop rhythm and crackles were heard throughout both lung fields. The initial diagnostic workup involved a complete blood count, urine analysis, electrocardiogram (ECG), and chest x-ray. Abnormal laboratory findings were as follows: creatinine level was $2.7 \mathrm{mg} / \mathrm{dl}$, potassium level was $5.9 \mathrm{mmol} / \mathrm{L}$, serum sodium level was $129 \mathrm{mmol} / \mathrm{L}$. He had hematuria and proteinuria, ECG revealed sinus tachycardia with 118 beats per minute. Chest x-ray revealed extensive bilateral interstitial pulmonary infiltrates, small bilateral pleural effusions, and cardiomegaly. Due to elevated serum creatinine levels and hematuria, patient evaluated by nephrologist. After a detailed examination, with his physical findings, blood counts (levels of ESR, CRP, cANCA) were high, initial diagnosis was vasculitis, especially Wegener's granulomatosis. Renal biopsy was performed and the result was compatible with Wegener's granulomatosis (Fig. 1). Transthorasic echocardiography was performed and revealed severely reduced left ventricular ejection fraction (EF) estimated around 25\%. Haemodialysis was performed. To determine if conduction pathway involvement was present or not, Holter ECG was recorded, and no rhythm problem was established.

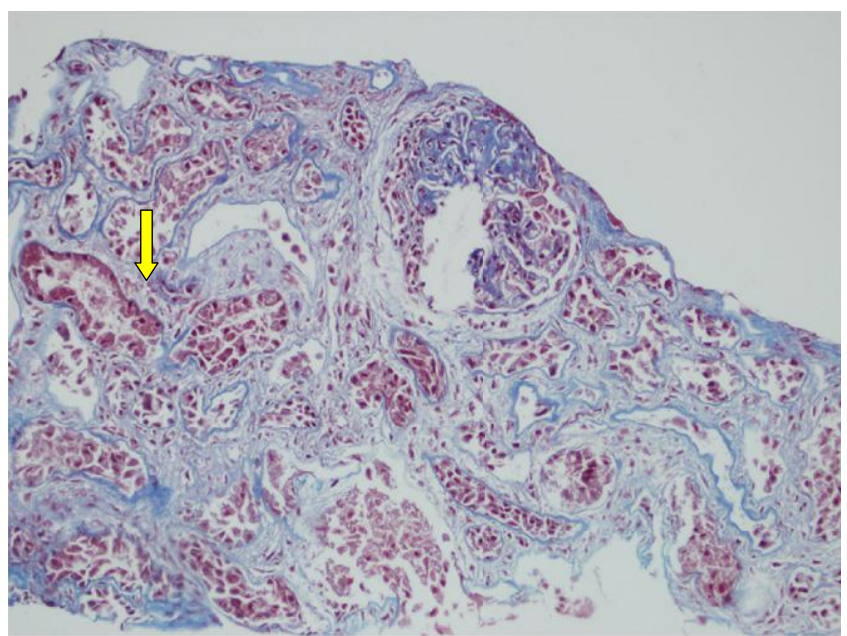

Fig 1. 


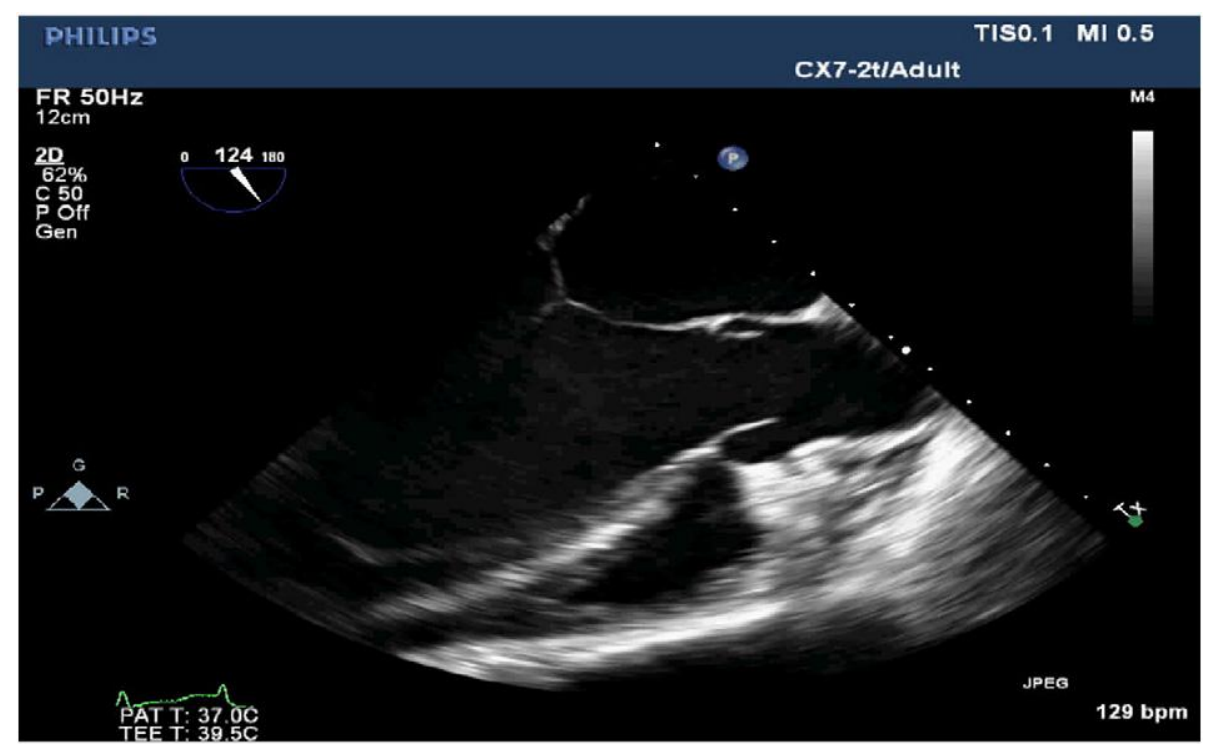

Fig. 2. Transesophageal echocardiography of the patient

Transeosephagial echocardiography showed no aortic involvement accept trivial aortic regurgitation (Figs. 2,3).

Cardiac MRI that was performed for myocarditis revealed dilated ventricules; late gadolinium enhancement (LGE) lesions involving LV myocardium and LGE lesions were found in subepicardial, midwall, and subendocardial LV myocardial layers, LVEF \%24.

We recommend coronary angiography but he refused it.

He was treated with furosemid, cyclophosphamide, and prostacyclin. He admitted to routine haemodialysis programme. After 1 month of follow-up, transthoracic echocardiography was performed again but no cardiac remission obtained. Medication continued for 3 months and then for the depressed systolic functions of left ventricle echocardiography repeated but no changes revealed. At the end of 6 months of follow-up period, his LVEF is the same as diagnosis period.

\section{Discussion}

Cardiac involvement of Wegeners's granulomatosis was first reported by Wegener in 1936 (3). Classical or generalised WG is characterised by necrotising granulomatous vasculitis of the upper and lower respiratory tract together with glomerulonephritis. Widespread disseminated vasculitis involving both small arteries and veins occurs to a greater or lesser degree as the disease progresses. A localised form of WG limited primarily to the upper and lower respiratory tracts has been described $(4,5)$. Despite histopathological diagnosis of WG, with autoantibodies against to circulatory neutrophilic cytoplasmic antigens, we can diagnose WG easily and early. WG must be kept in mind as the differential diagnosis of dilated cardiomyopathy, especially in the existence of pulmonary and renal pathologies. The clinical presentation of WG can be so diverse that the list of differential diagnoses is vast, ranging from infections (fungal, bacterial, and mycobacterial) to other vasculitides, including Henoch-Schönlein purpura, sarcoidosis, Behcet syndrome, and malignancies(6). Table 1 shows signs and symptoms and systemic involvement of WG $(6,7)$. Despite that involving the heart is well described, significant cardiac complications occurring during the course of the disease are rare (7). Pericarditis is the most common cardiac manifestation accounting for about $50 \%$ of cardiac diseases in Wegener's granulomatosis, which is asymptomatic in most of the cases, or may be manifested by chest pain and dyspnoea $(8,9)$. The overall mortality rate of Wegener's granulomatosis with cardiac

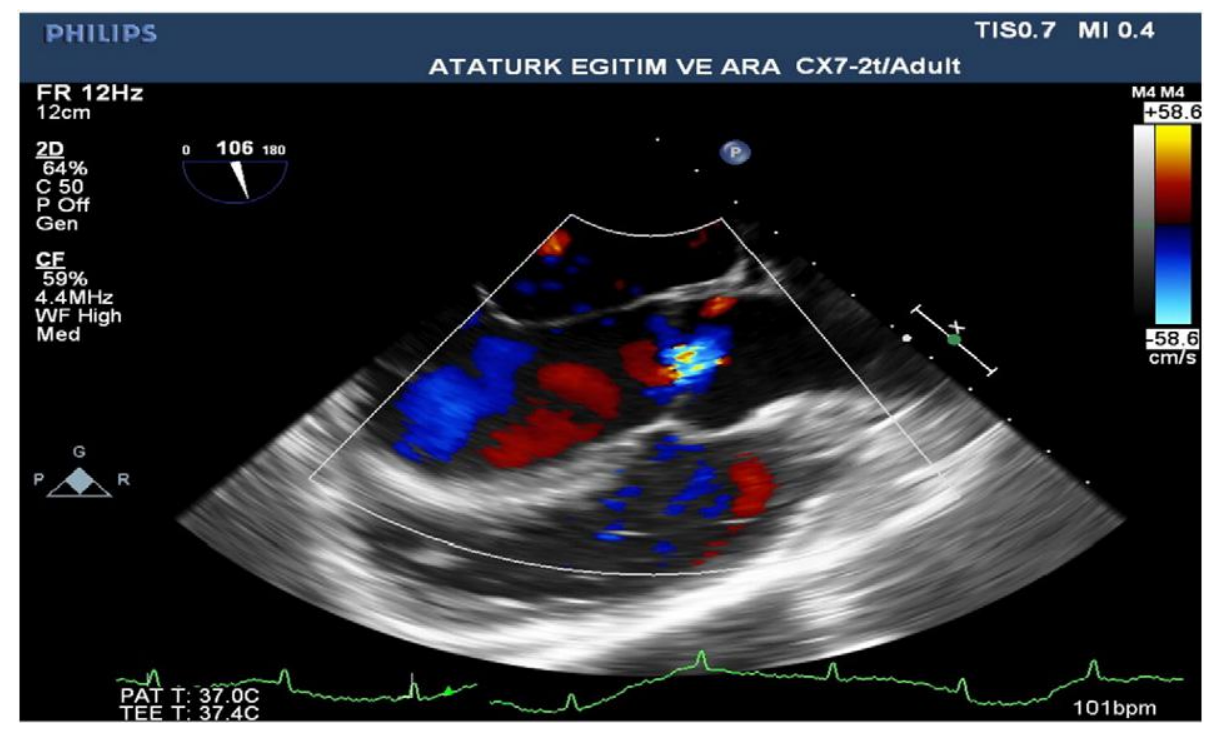

Fig. 3. Transesophageal echocardiography of the patient. 
Table 1

Signs and symptoms and systemic involvement of WG.

\begin{tabular}{llll}
\hline Signs and symptoms & $\%$ & & Systemic involvement \\
\hline Rhinorrhea and sinus pain & 94 & 100 & Respiratory tract \\
Fever & 78 & 83 & Renal \\
Anorexia and weight loss & 78 & 56 & Joints \\
Cough & 61 & 44 & Skin or muscle \\
Chest pain & 56 & 39 & Eye \\
Arthralgia & 56 & 39 & Middle ear \\
Skin lesions & 44 & 28 & Heart or pericardium \\
Otitis media & 39 & 22 & Nervous system \\
Eye symptoms & 39 & & \\
Hemoptysis & 22 & & \\
Arthritis & 22 & & \\
Neurological symptoms & 22 & & \\
\hline
\end{tabular}

involvement has been reported to be between 15 and 45\% (10). Cardiac manifestations are presented in Table $2(11,12)$.

In summary, Wegener's granulomatosis not infrequently affects the heart, particularly in more advanced cases of the disease, and may cause
Table 2

Cardiac manifestations in Wegener's granulomatosis.

\begin{tabular}{ll}
\hline -Pericarditis & -Conduction abnormalities \\
-Coronary arteritis & -Myocarditis \\
-Myocardial ischemia & -Noninfectious endocarditis \\
-Valvular regurgitation-stenosis & -Heart failure \\
\hline
\end{tabular}

clinically important complications. Pathologically, pericarditis, and coronary arteritis are the commonest manifestations. Clinically, evidence of pericarditis and its complications as well as supraventricular arrhythmias and varying degrees of heart block are the most common features. The advent of cANCA monitoring and the benefits of modem therapeutic approaches have resulted in many long-term survivors with previously severe Wegener's granulomatosis. Such patients not infrequently relapse with atypical presentations and cardiac involvement may therefore be seen more often in the future. 